\title{
Publisher's Note: Dynamical Birefringence: Electron-Hole Recollisions as Probes of Berry Curvature [Phys. Rev. X 7, 041042 (2017)]
}

\author{
Hunter B. Banks, Qile Wu, Darren C. Valovcin, Shawn Mack, Arthur C. Gossard, \\ Loren Pfeiffer, Ren-Bao Liu, and Mark S. Sherwin
}

(Q) (Received 6 November 2019; published 4 December 2019)

DOI: $10.1103 /$ PhysRevX.9.049902

This paper was published online on 21 November 2017 with an omission of a support statement in the Acknowledgments. The third sentence in the Acknowledgments on page 13 should read as "H. B. B., D. C. V., and M. S. S. were funded by NSF-DMR 1405964 and NSF-DMR 1710639." The Acknowledgments have been corrected as of 11 October 2019. The Acknowledgments are incorrect in the printed version of the journal.

Published by the American Physical Society under the terms of the Creative Commons Attribution 4.0 International license. Further distribution of this work must maintain attribution to the author(s) and the published articles title, journal citation, and DOI. 